\title{
ISRST: An Interest based Storytelling Model using Rhetorical Relations
}

\author{
Arturo Nakasone and Mitsuru Ishizuka \\ Dept. of Information and Communication Engineering. University of Tokyo. 7-3-1 Hongo, \\ Bunkyo Ku, Tokyo 113-8656, Japan \\ arturo@mi.ci.i.u-tokyo.ac.jp, ishizuka@i.u-tokyo.ac.jp
}

\begin{abstract}
Most storytelling model approaches consider stories formed by sequences of a particular type of event. These sequences are mostly constructed using the inherent temporal characteristic of each linked event and this limitation makes it difficult to adapt the models to other kinds of events. In order to develop a more generic model to create storytelling applications, we need to organize events using not only temporal relations, but also relations determined by the rhetorical context of those events. In this paper, we present ISRST, our proposal for a generic storytelling ontology model based on the organization of events using a subset of relations proposed by the Rhetorical Structure Theory and how narrative principles and user interest are applied to these relations to generate coherent stories.
\end{abstract}

Keywords: Interactive Storytelling, Ontology Model, RST, Rhetorical Relations, User Interest

\section{Introduction}

In a broad sense, stories are defined as unique sequences of events, mental states, or happenings involving human beings as characters or actors. The notion of story event is defined in several ways, each one to suit a particular way to deliver the intended message to the audience. Events can be represented as plain text [8,25,27], scripted individual character actions [9], story world states [15,16,29], or multimedia content [5,22]. Most storytelling models take these events and construct stories by creating sequences that depend highly on the inherent temporal property of such events. Even though the ways events are organized in these models are diverse and range from linear structures to web and modular structures [24], the tight coupling between content and temporality makes it very difficult to use or even adapt the models to other domains.

On the other hand, interactivity has occupied a central part in storytelling models due to the capacity of the computer to provide a much richer experience to the user by means of immersive interaction through the use of creative devices ranging from simple gamepads [22] to virtual rooms and objects embedded into mixed reality environments [14]. Most models deal with intrusive interaction in order to let users select their own way to experience the content of a story. Nevertheless, non intrusive 


\section{Arturo Nakasone and Mitsuru Ishizuka}

interaction in the form of emotion detection can be used to determine how this content is presented into the context of a whole narrative experience. A story experience may be seen as an emotional episode centered on a single most important emotion, which is interest, and extending with some degree of continuity throughout the entire story [30]. In this context, user interest can be used to formulate a special type of interaction in which the user shapes his or her experience of the story not in terms of intrusive manipulation of event content, but in terms of non intrusive manipulation of event sequences.

Therefore, in order to develop a more generic model to create storytelling applications, we focused the solution not on the content itself, but on the manner this content, in the form of events, is organized and how this organization is conveyed to the user in the context of an interesting narrative experience. In this paper, we present ISRST (Interactive Storytelling RST), our proposal for a generic storytelling ontology model based on the organization of events using a meaningful subset of relations proposed by the Rhetorical Structure Theory (RST) [17] and narrative principles applied to these RST relations combined with interest measurement to provide an interactive storytelling experience. This model was developed as an extension to our previous storytelling model SRST [19].

The rest of the paper is organized as follows. The next section will present related work on storytelling applications from the point of view of events and interactivity. Section 3 briefly discusses our previous approach and its limitations. Section 4 presents our current approach and how the limitations of the previous one were overcome. Section 5 discuss about the role of the interest emotion in our interactivity model. Finally, our plans for future research and a summary will conclude the paper.

\section{Related Work}

Even though storytelling applications were mostly oriented to text generation in the beginning, the range of applicability of storytelling has increased due to the ubiquitous presence of communication networks such as the Internet, and the availability of multimedia content. Most researchers assume a concept of "event” in order to organize the content of their applications, and, therefore, have developed storytelling engines to deliver such events using narrative techniques.

In Goal based applications [6,21], a goal event or events are established as the final outcome of the story. From a set of initial conditions, a story is unfolded by the sequence of events that are needed to reach such goal event or events. Planners and their variations are commonly used for these applications. Due to the nature of planners, events have to be annotated with a set of pre and/or post conditions. Nevertheless, some applications make use of special narrative functions that enforce narrative principles in the context of the whole story $[18,29]$. Therefore, event sequencing is determined not only by direct event links, but also by each event contribution to the overall storytelling experience.

State Transition based applications $[9,15,25,28]$ define events as states that specify the current situation in a particular point of the story. Bayesian Networks, Finite State 
Machines, and their variations are commonly used as storytelling engines. Therefore, events must be annotated to fit the requirements for nodes in the network or machine.

In Template based applications [10,26], events are selected to fit story templates and permutated to create new narrative experiences based on those templates. Since the story template constrains which and how events are presented, these events must be annotated to determine their role in the context of the whole template. Script based applications $[1,23,27]$ are a particular case in which stories are described using a high level language. The application, then, present the events in the way specified by such scripts. In some cases, narrative or dramatic effects are applied during the event transitions.

Semantically organized events using primarily RST have been used in applications that present multimedia content $[5,11,13]$, but most of them use only a very limited set of relations. Since rhetorically structured content was mainly used for organizational purposes, these applications do not deal with the narrative implications that rhetorical relations have in storytelling-like presentations.

In terms of interactivity, most models deal only with the intrusive aspect, without analyzing the emotional aspect of the story. Even though some models do not deal with interactivity at all $[1,23,11,19]$, most models deal with several kinds of intrusive interaction that goes from parameter specification $[5,10]$, and menu selection and interruptions $[29,27,13,7]$ to full user action multimedia processing $[21,28,6,18,15]$.

Even though the results obtained in these applications are impressive from the storytelling point of view, the cohesion between event content and storytelling engine makes the task of adapting these models to other domains extremely difficult. Therefore, a model in which event definition and sequencing are separated is necessary to guarantee its generic attribute.

\section{SRST: A Text Constrained Model using Rhetorical Relations}

We considered events in the world not to be isolated but interconnected through some kind of relation. Even though each event itself is meaningful in its content, the relations between them are what make them meaningful in the context of a story and semantic organization gave us not only a relationship of meaning, but also a relationship of temporality through the use of rhetorical extrapolations.

In our previous implementation [19], we made use of most of the rhetorical relations defined by RST, since our ontology corpus data was created using textual events, i.e. events that were defined as pieces of text presented through an agent. This allowed us to use the enormous amount of RST tools and data analysis to test our application. Nevertheless, the major problem with this first set of RST relations is that, since it was based entirely on text events, the creation of structures using other kinds of events, like graphics or animated scenes, was extremely difficult. RST was defined primarily for text organization in which an accurate distinction of the relations between pieces of text was the main objective. When we deal with content creation, this distinction does not present itself so accurate, since human beings do not tend to make use of such an extensive set of relation definitions in order to organize stories in their minds. 
The organization of events was based on a hierarchy composed by Stories, Scenes, and Acts. Since our application dealt mainly with text events, Acts were interpreted as Dialog Acts, or utterances an agent was to speak. The Act represented the primary class that organized events in a tree like structure, closely resembling the RST hierarchical structure. Nevertheless, this structure presented a serious challenge not only to content creation, but also to interactivity. In terms of content creation, a tree structure does not give the author enough flexibility, for instance, when he or she wants to define alternative beginnings and/or endings of a story. In terms of interactivity, a tree structure gives us only the possibility of one entry and one exit point in the story at any moment (i.e. the root of the tree or sub tree), which limits the ability of the engine to have more variety in terms of event selection.

Since events were to be presented by an Agent, the Agent class was defined inside the Act structure, and, through the use of the Role class, we assigned roles to each Agent based on which part of a relation this Agent could present. It is important to notice, however, that the Role class is useful as long as events are presented, but not when Agents are part of those events as actors.

In SRST, no user interaction was allowed. Therefore, the storytelling model defined a story template based on Bordwell and the notion of Conflict in a narrative [3]. This template properly defined the structure of a good narrative, but the presentation of events was constrained to the availability of text information at each level of the tree, making the use of the template irrelevant at levels when, for instance, only one relation was present.

For more information about our previous implementation, please see [19]. In the next section, we will discuss how we overcame these limitations in the current version and the new ontology specification that resulted from those improvements.

\section{ISRST: A Generic Event Interactive Model Approach}

In order to deal with the limitations of our previous model, we redesign the ontology taking into consideration the following issues:

\subsection{Appropriate Set of Rhetorical Relations}

Even though the way events are linked in any story are, in general, consistent with the definition of the complete set of RST relations, the first set made its use impractical for the purpose of event content creation, since most human beings make use of a more limited set of relations to construct and remember stories. In order to provide more flexibility to content authors, a reduced set of unambiguous relations was needed. This set was defined as a subset of the first set of RST relations used.

Most narratologists agree that the most important relation between events is the causal one (e.g. [4]). Nevertheless, we may argue that this is not the only relation that is taken into consideration when human beings try to create story patterns in their minds. As discussed by Ed Tan in [30], as we observe sequences of actions in a film, "the cognitive concern corresponds broadly to the assimilation of the action observed 
into a canonic narrative structure. This concern is also satisfied by a wealth of different relationships other than causal ones within the ultimate cognitive representation of the narrative”. Given the fact that a set of different types of relations help assimilate the content of a story, it is plausible to assume that the same set of relations may also be used to create such content. Therefore, we defined our set of relations taking into consideration their definition flexibility and the proper number of unambiguous relations that are needed to create generic patterns of stories.

For the current version of our model, the following relations were considered:

- Background: In this relation, one event $\mathrm{A}$ is referred as the context in which another event B happens. Event A happens to be located in the past of event B, but it does not necessarily entice a cause and effect relation.

- Cause: In this relation, one event is identified as the cause of another. The cause relation is one of the main relations in the ontology because it is the relation between events that people distinguish more clearly.

- Purpose: This relation reflects the necessity for one event to be shown before another can be shown. Even though it is in direct relation with the Cause relation, its use is different since it indicates a condition for advancing in the story, which will be discussed in Section 5.

- Result: This relation indicates that an event is shown as a direct consequence of another event. It is also linked to the Purpose relation, but has more immediate and final connotations. This relation is mostly used to indicate the display of final events during a story.

- Contrast: For any story to have narrative quality, it must show some kind of conflict between two or more events. Conflicts, implemented as contrasts, give stories an opportunity to enhance their audiences' comprehension and interest by creating narrative tension.

- Solutionhood: This relation provides a way to define how a Contrast relation will be solved.

- Elaboration: In this relation, an event is shown to give more details about another event.

- Evaluation: This relation provides a way to state a final conclusion about one event. It is helpful for authors to convey a final message or thought to the whole story.

- Sequence: This relation establishes a linear temporal link between two events. This relation is useful to enforce sequencing in stories, but it should not be used as the primary way to link events.

In the context of RST based analysis of texts, nucleus and satellite components of a relation are defined to identify which information is absolutely necessary (nucleus) to understand the text and which information is additional (satellite) and can be omitted without losing the meaning. By performing an extrapolation of these concepts into our event organization model, we let authors organize their content based on its importance to the development of the story. Nucleus events must always be presented in a story, but in the case of satellite events, the engine must decide which events are to be presented according to the user's interest in such story. In general, a good story must include the necessary nucleus events to fulfill its objective and enough satellite events to provide variety for the event selection engine. 


\subsection{The New Scene Structure}

Given previous arguments about the problems a tree structure may generate in terms of flexibility of content creation and story interactivity, we redefined the previously known Act structure (currently the Scene structure) in order to have an event organization based on the capacity of the structure to associate any two events by using a relation ${ }^{1}$. The most adequate Scene structure to fulfill this requirement was a directed graph in which each node represents either an Event or another Scene. A general scene structure implemented using XML is shown in Fig. 1.

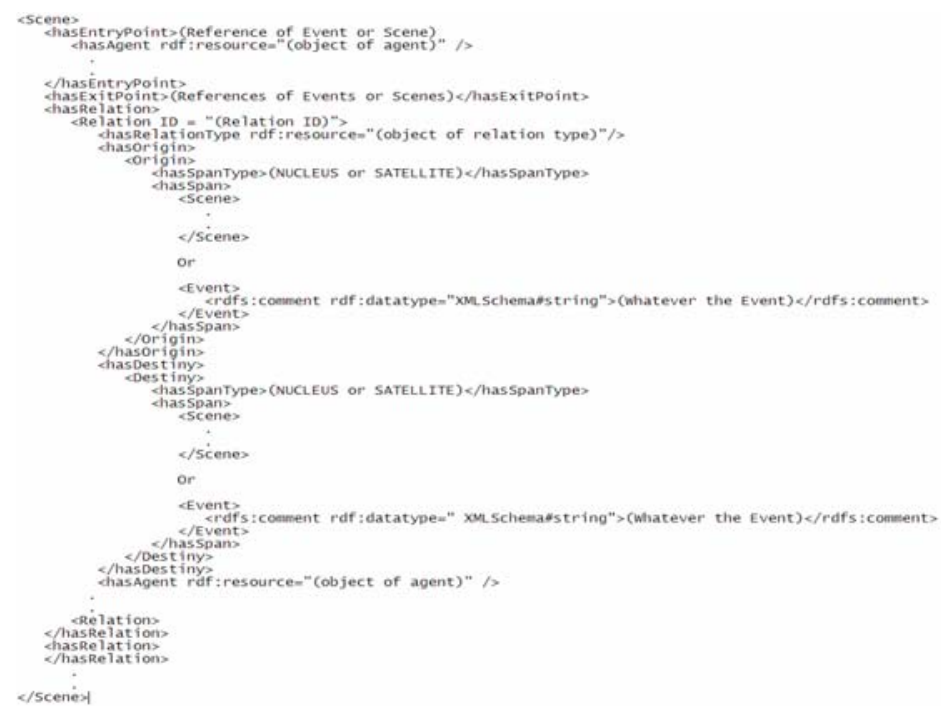

Fig. 1. General Schema of the New Scene Structure

The new definition of Scene shows a radically different approach to organize events, since its structure is basically composed of:

- An entry point, which determines the initial event (or scene) of the story and one or more exit points, which determine the possible events (or scenes) with which a story may end in this particular scene

- A set of relations that define how events are linked. It must be noted that a relation is composed of two events or scenes labeled as Origin and Destiny, as well as descriptions of the Agents that take part in the relation. This will be explained in detail in the following subsections.

${ }^{1}$ Since the relation between Scene and Act in our previous model caused some confusion because of the meaning of the word "Act" in other storytelling contexts, we adopted more conventional movie-style names. The previous Scene class is now the Episode class, and the previous Act class is now the Scene class. 


\subsubsection{The New Meaning of the Relation Class}

In our model we consider relations as unidirectional because they help us recognize which event constitutes the explained relation to which event. In this context, our representation of a relation between 2 events is given by the construction:

$\mathrm{A} \rightarrow \mathrm{R}(\mathrm{X}) \rightarrow \mathrm{B}$

Which is read as: The event $\mathrm{A}$ is the $\mathrm{R}$ of the event $\mathrm{B}$ applied to or regarding an agent $X$. In this case: $A$ is considered the Origin of the semantic relation $R(X)$ and $B$ is considered the Destiny. In our approach, the focus of the relation is centered in one or more agents, and this characteristic is defined as the agent's point of view.

\subsubsection{The Agent's Point of View (POV) in a Relation}

Events are constituted by Agents that perform certain actions either by themselves or in groups inside a determined Scene, which, for the purpose of this model, is assumed to obey the Aristotelian principles of unity of time, place, and action [24]. In many stories, characters' points of view help shape the story in the mind of the users, giving them a complete understanding of "the whole picture" inside the diegetic world they are voluntarily immersed in, by presenting several, if not all, of these viewpoints. An Agent's POV also help to study the preferences of the user towards a determined character based on interest and approach interactivity from a more personal and involving perspective. For this reason, we included in the model a property for each relation $\mathrm{R}$ which tells us to which agent or agents the relation is acting upon.

For example, if we have two events $\mathrm{A}$ and $\mathrm{B}$ which are related by the BACKGROUND relation, we could state that: $\mathrm{A} \rightarrow \mathrm{BACKGROUND}(\mathrm{X}) \rightarrow \mathrm{B}$ means that the event $\mathrm{A}$ is the background regarding the agent $\mathrm{X}$ of the event $\mathrm{B}$. With this approach, not only may we be able to have several background relations to one event, but also will give the narrative engine the opportunity to play with the interest of the user by selecting which relations applied to particular agents are shown or not.

\subsection{The New Storytelling Ontology Model}

Taking into consideration the limitations of the previous model and the solutions we devised to overcome those limitations, we redefined our OWL based ontology model to give content authors enough flexibility to create their stories and an event organization structure appropriate to deal with our interest based interactivity model. The main classes defined for this version of the ontology are:

- Concept: A Concept defines a specific topic that a presentation or part of it may refer about. This class remained unaltered from the previous version

- Event: An Event is defined as a single piece of meaningful information worthy of being presented. Due to the generic property of the model, an Event can now hold a reference to piece of text, video clip, image, game scene, character scripts, etc. 
- Relation: A Relation is a rhetorical binding between two entities, which refers to a specific rhetorical function.

- $\quad$ Agent: An Agent is an actor that takes part in a Relation and is affected by it.

- Origin and Destiny: This pair of classes defines the position of a determined entity in a Relation. An entity is defined as either an Event or a Scene.

- $\quad$ Scene: A Scene is defined as a graph-like structure composed of Relations. This class defines the minimum level of organization in which a story arises. A Scene is a recursive structure, which means that Origin and/or Destiny entities can reference an Event or another Scene object.

- Episode: An Episode is defined as a set of Scenes, which are grouped in the context of a single Concept. Nevertheless, for this version of the Ontology, only one Scene per Episode was considered.

- $\quad$ Story: A Story class frames the whole story and is composed of one or more Episodes.

The ExitPoint, EntryPoint, RelationType, and InfoType classes are auxiliary and are used to specify detailed information for the main classes. The complete diagram for the ontology model is shown in Fig 2.

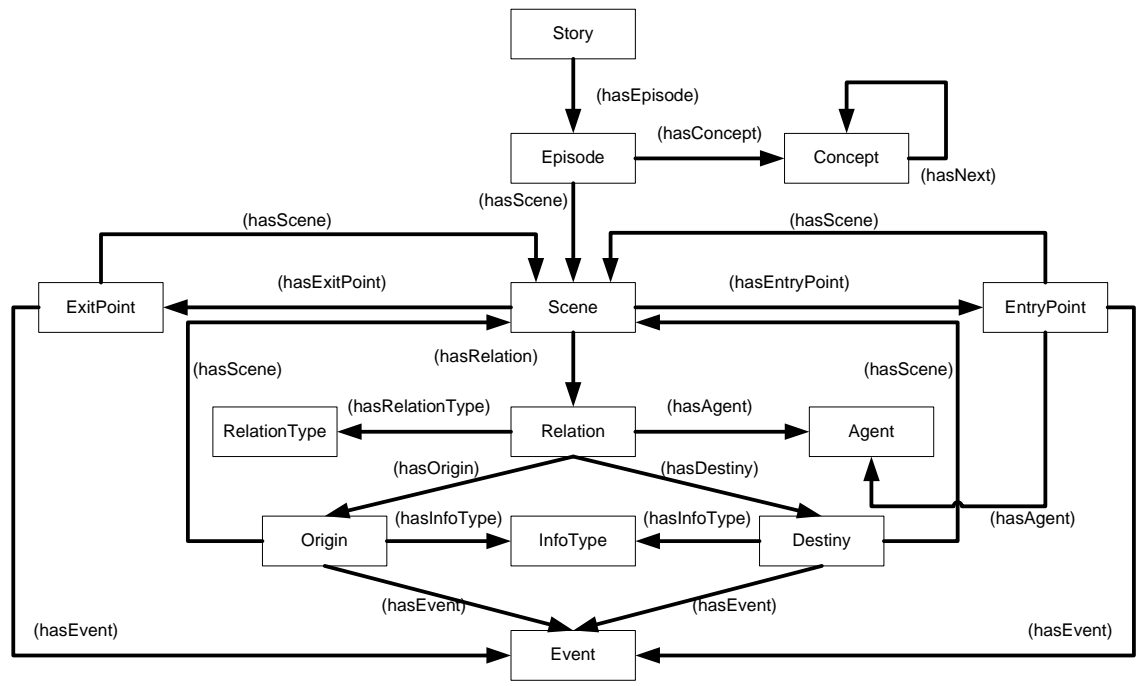

Fig 2. The ISRST Ontology Model

In the next section, we will talk about how a story is constructed based on the elements described above, the narrative rules that are applied to create generic story templates and the role that users' interest plays in the shaping of the overall narrative experience. 


\section{Interest Interaction in the Narrative Engine}

Interactivity in storytelling can be treated in several different ways. Some models regard interactivity as simple as menu choices during the story and others use more complex hardware to give us a true immersive experience. This view of interactivity concentrates on what users can do and manipulate inside the environment of the story, but not on the layout of the story itself. Even though users have a lot of freedom in most systems like RPGs (Role Playing Games) to shape their experience as they wish, it can be argued that almost none of these systems attempt to map these interactions to the actual feeling of interest of the user on how he or she wants the story to be shaped.

The view of interactivity that our model addresses concerns the fact that users can "select" which events to see in the story based on their interest as the story progresses. Interest, being a long lasting emotion, can shape the layout of the story in terms of what the user believes to feel at a particular moment. Even though a story can elicit several kinds of emotions, interest remains as the sole emotion that can determine whether a story is following the right path from the users' perspective ${ }^{2}$.

Therefore, in order to construct a story, our engine implements three concepts that are applied simultaneously during the construction process. These concepts will be detailed in the following subsections.

\subsection{The Concept of Advancing the Story}

Since most of the rhetorical relations used in the model do not clearly imply a temporal relation between the events that they relate, we defined a method to linearize the events in the story construction process. Given a relation set formed by:

$\mathrm{A} \rightarrow \mathrm{R}(\mathrm{X}) \rightarrow \mathrm{B}$, we defined the concept of Advancing the Story through the Relation $\mathrm{R}$ if the story, being located at the event/scene $\mathrm{B}$ goes to show the contents of the event/scene A through the relation $\mathrm{R}(\mathrm{X})$. In other words, at any moment in the presentation, we analyze all the incoming relations to the current event, and move to the origin of the relation that defines the event that will be shown next. This concept may seem contradictory since we seem to be going backwards in the relation, but we selected this way of processing because:

- It gives a unified model for creating relations in a presentation.

- Not all relations follow the pattern of advancing described above. For example, the CAUSE relation indicates that event $\mathrm{A}$ is the cause of event $\mathrm{B}$, but does not necessarily imply that the story moves forward when going to A. These special cases are dealt accordingly, but if we were to specify any relation by its pseudotemporal attribute, we may end up having a non-uniform model for content creation. From our point of view, it is much better if these particularities are dealt by the engine and not by the content author.

\footnotetext{
${ }^{2}$ Since interest measurement is out of the scope of this model, the measure of interest is done in a direct way (by asking users directly), though as non intrusive as possible.
} 


\subsection{The Concept of Relation Narrative Template}

In order to integrate the concept of narrative in the new Scene structure, we made a distinction among the several relations, so we could determine which ones belong either exclusively or generally to each phase of a story. Therefore, we took the narrative template from our previous version of the ontology and categorized the relations according to this template. Nevertheless, we also took into consideration the fact that each phase must allow the proper relations for the story to advance. Our implemented narrative template contemplates the following phases:

- Story Introduction: Only the BACKGROUND relation defines the introduction of a story. The complexity of an introduction phase will come from the fact that a background relation can link either an event or a full scene structure, which may have its own background.

- Story Conflict: Only the CONTRAST relation is considered as the initiator of conflict, or the crisis introducer. Again, the contrast relation can associate either 2 events or 2 scenes for more complex constructions.

- Story Resolution: Only the SOLUTIONHOOD relation is considered as the initiator for the path to climax in the story

- Story Conclusion or Coda: Only the EVALUATION relation is considered as the initiator for the final conclusion or epilogue of the story

The other relations in the set are called Generic Relations. They can be included in any phase of the story, and be used to define very complex structures in terms of relations inside each phase. These relations are: SEQUENCE, ELABORATION, PURPOSE, RESULT, and CAUSE.

\subsection{The Concept of Interest Based Agent Preference}

When the engine faces the alternative of selecting an event among two or more satellite events, the registered interest values from the user determine the choice by associating such values with the characters that take part in the relations that link the satellite choices. In other words, if we have two or more satellite relations from where to choose at a certain point in the story, our model defines the concept of Interest Based Agent Preference (IBAP) thorough the following criteria:

- A relation $R_{1}\left(X_{1}, X_{2}, . ., X_{n}\right)$ has interest precedence over a relation $R_{2}\left(Y_{1}, Y_{2}, \ldots\right.$, $Y_{n}$ ) if the sum of interest averages for $X_{1}, X_{2}, . ., X_{n}$ is greater than the sum of averages for $Y_{1}, Y_{2}, \ldots, Y_{n}$.

- When we have two or more satellite relations which have the same average sum, a relation will be chosen based on narrative template precedence. 


\section{Conclusion and Future Work}

In this paper, we have presented our current version for a generic storytelling ontology model based on the organization of events using a meaningful subset of the relations proposed by RST and user interest based interactivity. Now, we are working on an implementation of an application that will present story content in the form of images (cartoon style), that can be deployable on the Web. This application is based on our previous prototype using Kaon2 [12], and it will be used to conduct studies on how users' interest affects event sequencing in a story and vice versa.

Even though there are several studies on how to measure user's interest through the use of either bio-signals to determine emotion [20] or gaze tracking [2], we will use a rather direct approach to measure the interest of a user, which is by asking through a simple slider control his or her level of interest in the current event of the story. Even though this way of measuring interest can be considered obtrusive, in the particular case of the interest emotion, it was proved that it can be measured by means of self report methods, due to its dominant property in the course of a story presentation [30].

\section{References}

1. André, E., Concepcion, K., Mani, I., van Guilder, L. Autobriefer: A System for Authoring Narrated Briefings. Multimodal Intelligent Information Presentation , Oliviero Stock and Massimo Zancanaro , 143-158, Springer, 2005

2. Bee, N., Prendinger, H., Nakasone, A., André, E., and Ishizuka, M. AutoSelect: What You Want Is What You Get. Real-time processing of visual attention and affect. Proceedings International Tutorial and Research Workshop on Perception and Interactive Technologies (PIT-06), Springer LNAI 4021, Kloster Irsee, Germany, 2006, 40-52.

3. Bordwell, D. Narration in the Fiction Film. London Routledge, 1986

4. Branigan, E. Narrative Comprehension and Film. London Routledge, 1992.

5. Callaway, C., Not, E., Novello, A., Rocchi, C., Stock, O., Zancanaro, M. Automatic Cinematography and Multilingual NLG for Generating Video Documentaries. In Artificial Intelligence, vol. 165, June 2005, 57-89

6. Cavazza M., Charles F., and Mead S.J. Interacting with virtual characters in interactive storytelling. In Proceedings First Conference on Autonomous Agents and Multiagent Systems (AAMAS-02), New York, 2002. ACM Press, 318-325

7. Crawford, C. Chris Crawford on Interactive Storytelling. New Riders 2005.

8. Figa, E. and Tarau, P. Story Traces and Projections: Exploring the Patterns of Storytelling. In N. Braun and U. Spierling, editors. First International Conference on Technologies for Interactive Digital Storytelling and Entertainment (TIDSE 2003), Darmstadt, Germany, March 2003.

9. Gebhard, P., Kipp, M., Klesen, M., Rist, T.: Authoring scenes for adaptive, interactive performances. The Second International Conference on Autonomous Agents and Multiagent Systems (AAMAS-03). 725-732

10. Gervas P., Diaz-Agudo B., Peinado F, and Hervas R. Story plot generation based on CBR. Journal of Knowledge Based Systems 18 (2-3), 2005, 235-242.

11. Geurts J., Bocconi S., van Ossenbruggen J., and Hardman L. Towards Ontology-Driven Discourse: From Semantic Graphs to Multimedia Presentations. In Proceedings of the International Semantic Web Conference 2003, 597-612.

12. Kaon2 Reasoning Engine. http://kaon2.semanticweb.org/ 
13. Little, S., Geurts, J., Hunter, J. Dynamic Generation of Intelligent Multimedia Presentations through Semantic Inferencing, In Proceedings of the 6th European Conference on Research and Advanced Technology for Digital Libraries, September 2002.

14. Lindinger, C., Haring, R., Hortner, H., Kuka, D., Kato, H. Mixed Reality Installation 'Gulliver's World': Interactive Content Creation in Nonlinear Exhibition Design. Third International Conference on Technologies for Interactive Digital Storytelling and Entertainment, TIDSE 2006, December 4-6, 2006, Darmstadt, Germany, 312-323.

15. Magerko, B. and Laird, J.E. Building an Interactive Drama Architecture. First International Conference on Technologies for Interactive Digital Storytelling and Entertainment, 2003. Darmstadt, Germany, 226-237.

16. Magerko, B. and Laird, J.E. Mediating the Tension Between Plot and Interaction. AAAI Workshop Series: Challenges in Game Artificial Intelligence, 2004. San Jose, California, pp. 108-112.

17. Mann, W., and Thompson S. Rhetorical structure theory: A theory of text organization. Marina del Rey, CA: Information Sciences Institute, 1987.

18. Mateas, M., Stern, A. Façade: An Experiment in Building a Fully-Realized Interactive Drama; Game Developers Conference, Game Design Track, March 2003

19. Nakasone A., Ishizuka M. SRST: A Storytelling Model using Rhetorical Relations. Third International Conference on Technologies for Interactive Digital Storytelling and Entertainment, TIDSE 2006, December 4-6, 2006, Darmstadt, Germany, 127-138.

20. Nakasone A., Prendinger H., and Ishizuka M. Emotion recognition from electromyography and skin conductance. The Fifth International Workshop on Biosignal Interpretation (BSI-05), Tokyo, Japan, 2005, 219-222.

21. Riedl, M.O., Saretto, C.J., Young, R.M. Managing interaction between users and agents in a multi-agent storytelling environment. The Second International Joint Conference on Autonomous Agents \& Multiagent Systems, AAMAS 2003, July 14-18, 2003, Melbourne, Victoria, Australia, Proceedings. ACM 2003. 741-748

22. Rober, N., Huber, C., Hartmann, K., Feustel, M., Masuch, M. Interactive Audiobooks: Combining Narratives with Game Elements. TIDSE 2006, December 4-6, 2006, Darmstadt, Germany, 358-369.

23. Sgouros, N.M., Papakonstantinou, G., Tsanakas, P. Dynamic Dramatization of Multimedia Story Presentations. ACM International Conference on Intelligent User Interfaces (IUI97), Orlando, FL, USA

24. Sheldon, L. Character Development and Storytelling for Games. Course Technology PTR, 2004

25. Silva A., Guilherme R., and Paiva A. Tell me that bit again... Bringing Interactivity to a Virtual Storyteller. In Proceedings of the 2nd International Conference on Virtual Storytelling - ICVS 2003, 146-154.

26. Sobral, D., Machado, I., Paiva, A. Managing authorship in plot conduction. In Virtual Storytelling: Using Virtual Reality Technologies for Storytelling. Springer 2003.

27. Sumi, K., Tanaka, K. Automatic Conversion from E-content into Animated Storytelling, Entertainment Computing-ICEC2005, Springer Lecture Note in Computer Science, 24-35 (September, 2005).

28. Swartout, W., Hill, R., Gratch, J., Johnson, W.L., Kyriakakis, C., Labore, K., Lindheim, R., Marsella, S., Miraglia, D., Moore, B., Morie, J., Rickel, J., Thiebaux, M., Tuch, L., Whitney, R. Toward the Holodeck: Integrating Graphics, Sound, Caracter and Story in Proceedings of the 5th International Conference on Autonomous Agents, 2001

29. Szilas, N. IDtension: a narrative engine for Interactive Drama; 1st International Conference on Technologies for Interactive Digital Storytelling and Entertainment (TIDSE 2003), Germany, March 24-26 2003.

30. Tan, E. Emotion and the Structure of Narrative Film. Lawrence Erlbaum Associates, Mahwah, NJ, 1996 